\title{
Um estudo sobre os papéis propostos no XS-CMM em uma Empresa de Pequeno Porte
}

\author{
Luiz Fernando de Oliveira Silva ${ }^{1}$, Sandra C. P. Ferraz Fabbri ${ }^{1}$ \\ ${ }^{1}$ Departamento de Computação - Universidade Federal de São Carlos \\ Rodovia Washington Luís, Km 235 Cx. Postal 676, 13565-905 \\ São Carlos, SP, Brasil \\ \{luiz,sfabbri\}@dc.ufscar.br
}

\begin{abstract}
This paper describes an experience of using XS-CMM in a small organization, emphasizing the sharing and charging the roles proposed in this model, in relation to the roles proposed in SW-CMM. Although the target of this model is the small organization, the experience showed that the roles and activities established in the organization define and require another relationships not predicted in the model.

Resumo. Este artigo descreve uma experiência de aplicação do XS-CMM em uma empresa de pequeno porte, dando-se ênfase ao acúmulo $e$ compartilhamento dos papéis propostos nesse modelo, em relação aos papéis definidos no SW-CMM. A experiência mostrou que, embora o modelo tenha como alvo esse tipo de empresa, ainda assim, os cargos e funções estabelecidos na empresa definem e exigem outros tipos de relacionamentos não previstos no modelo.
\end{abstract}

\section{Introdução}

A preocupação com a qualidade do processo de software tem sido cada vez maior, seja por questões de necessidade e competitividade, seja por conscientização e tomada de decisão, como é o caso da empresa tratada neste artigo. Dentre os modelos de qualidade de processo propostos na literatura, o SW-CMM [1] parece ser ainda o mais utilizado no mercado [2]. No entanto, esse modelo foi criado visando empresas de grande porte e, em decorrência disso, existe muita dificuldade em implantá-lo em empresas de pequeno porte, devido às adaptações necessárias. Assim, notam-se alguns trabalhos na literatura $[3,4,5,6,7]$ que têm o objetivo de relatar experiências ou propor diretrizes que auxiliem essa adaptação.

Alguns desses trabalhos são de Orci [5,6,7], que propuseram três variantes para o modelo SW-CMM, que estão relacionadas a empresas de pequeno porte, dependendo de suas características e, principalmente, do número de funcionários. Um desses modelos é o XS-CMM (eXtra Small CMM) [5], cujas características, por se adequarem à empresa em questão, foi o modelo usado neste contexto. Neste artigo dá-se ênfase justamente a um dos principais aspectos que dificultam a adequação do SW-CMM em empresas menores, que é a quantidade reduzida de funcionários que ela tem e que impossibilita a atribuição dos vários papéis existentes nesse modelo.

O artigo está organizado da seguinte forma: na Seção 2 dá-se uma breve caracterização da empresa abordada no estudo; na Seção 3 descrevem-se as principais características do modelo XS-CMM; na Seção 4 apresenta-se o estudo realizado, dando- 
se ênfase à questão dos papéis envolvidos com os modelos SW-CMM e XS-CMM; na Seção 5 comentam-se as lições aprendidas e na Seção 6 as conclusões.

\section{A Empresa do estudo de caso}

A empresa na qual o estudo de caso foi realizado é uma prestadora de serviços que atua no segmento de conexão à internet, desenvolvimento de sistemas para Web e serviços de telecomunicações. Ela serve a cidade e região de São Carlos, interior de São Paulo, e possui diversas unidades de negócios. O setor de desenvolvimento de sistema Web, chamado ProdWeb, foi o ambiente do estudo de caso deste trabalho.

O Prodweb está estruturado da seguinte maneira: possui um coordenador geral para gerenciamento e acompanhamento dos projetos; uma equipe de $\mathrm{P} \& \mathrm{D}$, responsável por pesquisas e desenvolvimento de novas tecnologias e algumas equipes de desenvolvimento. Além disso, os consultores comerciais, que também estão alocados nesse setor, são responsáveis pelo levantamento de requisitos. Ao todo estão envolvidos cerca de 15 funcionários no processo de software do Prodweb, o que caracteriza esse ambiente de estudo como sendo uma "Empresa Muito Pequena", de acordo com a classificação [5,8]. No contexto deste trabalho essa empresa será referenciada com o termo "Empresa de Pequeno Porte".

\section{A abordagem aplicada: XS-CMM}

O XS-CMM - eXtra Small CMM - é um dos modelos propostos por Orci [5], adaptados do SW-CMM e direcionados para empresas que não são de grande porte. Além desse modelo, Orci propôs outros dois: XXS-CMM [6] (eXtra eXtra Small CMM) e S-CMM [7] (Small CMM). O XXS-CMM corresponde à Micro Empresa (até 2 funcionários), o XS-CMM à Empresa Muito Pequena (de 3 a 15 funcionários) e o S-CMM à Pequena Empresa (mais de 15 funcionários). Em outros estudos Orci et al. [9,10] propuseram adaptações e suplementos para acompanhamento e avaliação da implantação do SWCMM nessas categorias de empresas, de acordo com os modelos citados.

Considerando-se as características da empresa em questão, mencionadas na Seção 2, o modelo adequado a ela é o XS-CMM, cujas adaptações podem ser sintetizadas no seguinte: i) Adaptação das KPA's do SW-CMM, ii) Acúmulo de Papéis do SW-CMM, iii) Compartilhamento de funções entre os papéis, iv) Outros Recursos.

i) Adaptação das KPA's do SW-CMM [5]: Orci reestruturou o modelo SW-CMM adequando cada KPA (Key Process Area), por meio de suas práticas chaves, à realidade das Empresas de Pequeno Porte. A Tabela 1 apresenta a quantidade dessas práticas nos dois modelos. Como pode ser observado, a KPA Gerenciamento de Subcontratação de Software foi excluída e houve uma redução de Práticas Chaves nas KPA's Acompanhamento e Supervisão de Projeto e Garantia de Qualidade de Software. Além disso, em alguns casos, a descrição dessas práticas chaves foi alterada, em decorrência de alguns papéis que não existem no ambiente de Empresas de Pequeno Porte, o que será comentado a seguir.

ii) Acúmulo de Papéis do SW-CMM: segundo Orci, no contexto de uma Empresa de Pequeno Porte não é possível existir alguns papéis presentes no SW-CMM, em virtude do número reduzido de funcionários [5]. Portanto, o autor propõe o acúmulo de papéis apresentado na Tabela 2. Esse acúmulo significa que as tarefas que eram realizadas por um determinado papel do SW-CMM agora são feitas pelo correspondente papel do XSCMM. 
Tabela 1 - Quantidade de Práticas Chave do SW-CMM e XS-CMM

\begin{tabular}{|c|c|c|c|c|}
\hline & \multicolumn{2}{|l|}{ SW-CMM } & \multicolumn{2}{|l|}{ XS-CMM } \\
\hline & KPA's & $\begin{array}{l}\text { Práticas } \\
\text { Chaves }\end{array}$ & KPA's & $\begin{array}{l}\text { Práticas } \\
\text { Chaves }\end{array}$ \\
\hline & Gestão de Requisitos & 12 & Gestão de Requisitos & 12 \\
\hline & $\begin{array}{l}\text { Planejamento de Projeto de } \\
\text { Software }\end{array}$ & 25 & $\begin{array}{l}\text { Planejamento de Projeto de } \\
\text { Software }\end{array}$ & 25 \\
\hline & $\begin{array}{l}\text { Acompanhamento e Supervisão de } \\
\text { Projeto de Software }\end{array}$ & 24 & $\begin{array}{l}\text { Acompanhamento e Supervisão } \\
\text { de Projeto de Software }\end{array}$ & 23 \\
\hline & $\begin{array}{l}\text { Gestão de Subcontratação de } \\
\text { Software }\end{array}$ & 22 & ---- & 0 \\
\hline & Garantia da Qualidade de Software & 17 & $\begin{array}{l}\text { Garantia da Qualidade de } \\
\text { Software }\end{array}$ & 15 \\
\hline & $\begin{array}{l}\text { Gestão de Configuração de } \\
\text { Software }\end{array}$ & 21 & $\begin{array}{l}\text { Gestão de Configuração de } \\
\text { Software }\end{array}$ & 21 \\
\hline TOTAL & 6 & 121 & 5 & 96 \\
\hline
\end{tabular}

Em relação a esse acúmulo de papéis, por exemplo, Orci considera que uma Empresa de Pequeno Porte não possui recursos para manter o Grupo de Gerenciamento de Contrato (GGC) e portanto, a função desse grupo é acumulada pelo papel Vendas e Marketing (VM) no XS-CMM.

Tabela 2 - Relacionamento entre os papéis do SW-CMM e XS-CMM [5]

\begin{tabular}{|l|l|}
\hline \multicolumn{1}{|c|}{ Papéis do SW-CMM } & \multicolumn{1}{c|}{ Papéis do XS-CMM } \\
\hline $\begin{array}{l}\text { Representação do Grupo de Garantia de } \\
\text { Qualidade SW do cliente (GQSC) }\end{array}$ & $\begin{array}{l}\text { Representação do Grupo de Garantia de SW do } \\
\text { cliente (GQSC) }\end{array}$ \\
\hline $\begin{array}{l}\text { Vendas e Marketing (VM) } \\
\text { Grupo de Gerenciamento de Contrato (GGC) }\end{array}$ & Vendas e Marketing (VM) \\
\hline $\begin{array}{l}\text { Gerente de Projeto (GP) } \\
\text { Grupo de Estimativa de Software (GETS) }\end{array}$ & Gerente de Projeto (GP) \\
\hline Gerente Sênior (GS) & Gerente Sênior (GS) \\
\hline $\begin{array}{l}\text { Grupo de Gerenciamento de Config. SW (GCS) } \\
\text { Grupo de Controle de Config SW (GCCS) }\end{array}$ & Grupo de Gerenciamento de Config. SW (GCS) \\
\hline $\begin{array}{l}\text { Grupo de Engenharia de Software (ES) } \\
\text { Grupo de Suporte à Documentação (GSD) }\end{array}$ & Grupo de Engenharia de Software (ES) \\
\hline Gerente de Software (GSW) & Gerente de Software (GSW) \\
\hline Grupo de Garantia de Qualidade SW (GQS) & Grupo de Garantia de Qualidade SW (GQS) \\
\hline $\begin{array}{l}\text { Grupo de Engenharia de Sistemas (GES) } \\
\text { Grupo de Engenharia de Hardware (GEH) }\end{array}$ & Grupo de Engenharia de Sistemas (GES) \\
\hline Grupo de Teste de Sistemas (GTS) & Grupo de Teste de Sistemas (GTS) \\
\hline
\end{tabular}

iii) Compartilhamento de funções entre os papéis: em decorrência do acúmulo de papéis, quando estes são assumidos por um único funcionário, pode haver uma sobrecarga de funções. Portanto, Orci [5] propõe o compartilhamento das funções entre os papéis, conforme apresentado na Figura 1, para que as pessoas na Empresa de Pequeno Porte não fiquem sobrecarregadas em decorrência disso. Nessa figura os círculos representam os papéis; as linhas que ligam os círculos indicam que os papéis podem ser compartilhados entre os funcionários que estão assumindo os referidos papéis e o círculo tracejado representa o grupo de garantia de qualidade de software do cliente, caso exista. Assim, de acordo com a Figura 1, por exemplo, o Gerente Sênior (GS) pode compartilhar suas funções com o Grupo de Engenharia de Software ou com Vendas e Marketing e vice-versa. 


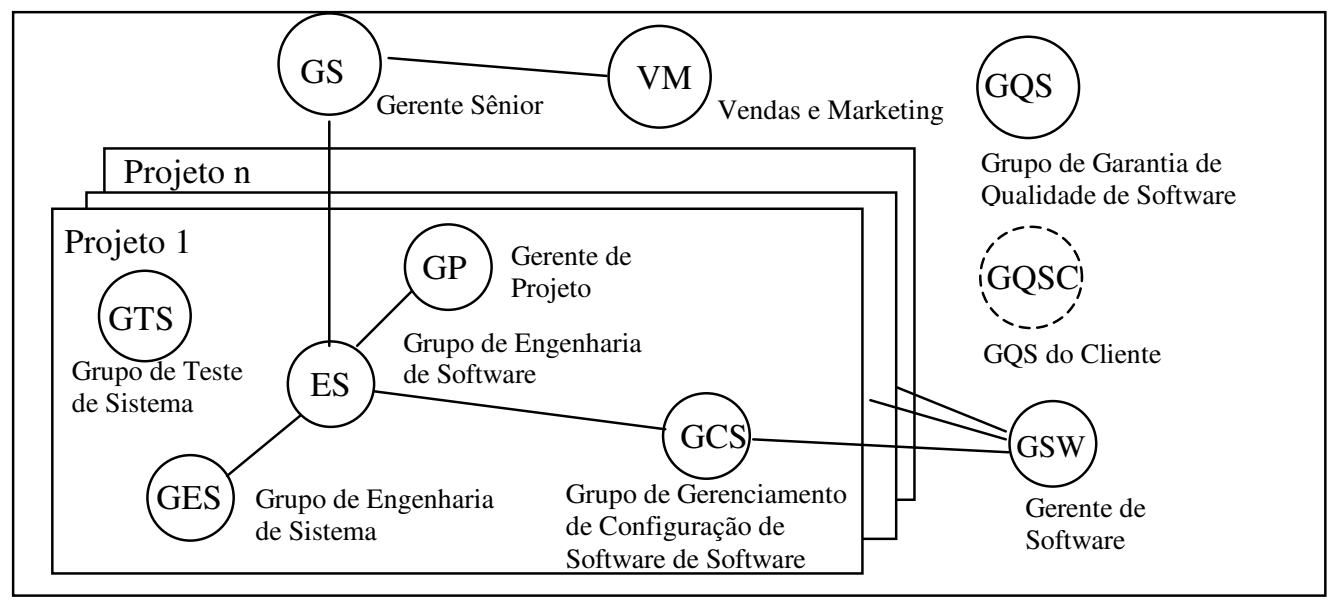

Figura 1- Compartilhamento de funções entre os papéis do XS-CMM [5]

iv) Outros Recursos: Orci et al $[9,10]$ desenvolveram alguns suplementos para auxiliar a implantação do XS-CMM nas Empresas de Pequeno Porte. Esses suplementos abrangem a adaptação de documentos, questionários para auto-avaliação, checklists para acompanhamento da implantação do XS-CMM e medições.

\section{Melhorias realizadas \& XS-CMM}

Nos últimos anos, a empresa do estudo de caso vem investindo na melhoria de seu processo de software e foi alvo de estudo no trabalho de Tavares [12] o qual teve sua continuidade no contexto deste trabalho. Tavares realizou um diagnóstico da empresa em relação ao nível 2 do SW-CMM, definiu o processo padrão de desenvolvimento de software, planejou a melhoria e criou o plano de ação para o início das atividades dessa melhoria, de acordo com diretrizes previamente definidas.

O início deste trabalho foi caracterizado pela realização de algumas reuniões para conhecimento da empresa, identificação dos efeitos do trabalho de Tavares e definição da equipe de qualidade que seria responsável pela implantação do programa de melhoria do processo de software. Essa equipe foi formada por alguns integrantes da equipe de desenvolvimento, o gerente da empresa e a equipe acadêmica. Embora o trabalho de Tavares tenha sido baseado no SW-CMM, uma vez identificado e estudado o modelo XS-CMM, decidiu-se buscar nesse modelo algumas diretrizes que pudessem facilitar a melhoria do processo, dada a correspondência existente entre esse modelo e a empresa em questão. No contexto deste trabalho, as atividades realizadas podem ser agrupadas em três decisões principais, sendo que neste artigo exploram-se somente as ações que foram inspiradas no XS-CMM, mais particularmente, o estudo realizado em relação ao acúmulo e compartilhamento dos papéis:

Decisão 1 - alteracão do processo padrão: o processo estabelecido no trabalho de Tavares [12] foi alterado por serem constatadas várias dificuldades na sua utilização, por parte da equipe de desenvolvimento.

Decisão 2 - alteracão da estratégia de implantacão das melhorias: inicialmente, a abordagem de implantação das melhorias seguia as KPA do XS-CMM, como é relatado nos trabalhos de Orci e Laryd [9] e Kelly e Culleton [13], e que estava recomendada no Plano de Ação estabelecido no trabalho de Tavares [12]. No entanto, em decorrência dos poucos resultados observados, decidiu-se alterar a abordagem, priorizando-se os principais problemas que a empresa vivenciava diariamente e propondo soluções de 
acordo com o especificado na(s) KPA relacionada(s) a eles. Nessa etapa elaboraram-se dois formulários, ambos inspirados nos documentos relacionados no item iv da Seção 3:

a) Formulário de Priorização: para documentar os problemas identificados no dia a dia da empresa, o qual contém campos para descrição do problema, as pessoas envolvidas na sua solução, os participantes da reunião e os resultados alcançados e lições aprendidas.

b) Checklist de Acompanhamento: para acompanhar a implantação de cada prática chave da(s) KPA associada(s) ao problema identificado, o qual foi baseado em [10] e registra se as práticas chaves foram parcialmente ou totalmente atendidas, sendo que o "totalmente" representa o que foi estabelecido como alvo pela própria empresa.

Decisão 3 - implantacão de duas melhorias: Melhoria-1 relacionada aos erros que existiam na elaboração dos produtos de software e Melhoria-2 relacionada às políticas de levantamento de requisitos e elaboração dos cronogramas.

a) Melhoria-1: com base nas práticas chaves da KPA de Garantia de Qualidade de Software (item i, Seção 3) estabeleceram-se procedimentos para dirimir os problemas priorizados em relação aos produtos intermediários gerados durante o processo de desenvolvimento de software. Foram então definidos procedimentos para criação de layout de páginas e checklists baseados nesses procedimentos.

b) Melhoria-2: com base nas práticas chaves das KPA's Gerenciamento de Requisitos e Planejamento de Software (item i, Seção 3) estabeleceram-se as instruções para as políticas de levantamento de requisitos e de elaboração dos cronogramas e determinação de prazos. Foi ao tentar realizar esta melhoria na empresa que a questão dos papéis, abordada com ênfase no modelo XS-CMM, mostrou-se relevante. Percebeu-se que para definir tais políticas era necessário ter claramente definidos os papéis de cada profissional envolvido nessa atividade e nas demais relacionadas ao processo de desenvolvimento de software. Dessa forma, realizou-se um estudo da proposta de Orci [5] sobre acúmulo de papéis e compartilhamento de funções (item ii e iii, Seção 3), no contexto da empresa.

O primeiro passo foi criar uma tabela com as funções de cada papel [5,11], para relacioná-las com as funções de cada cargo existente na empresa, conforme apresentado na Tabela 3.

Quando alguma função de algum papel não era realizada por algum cargo, anotava-se o cargo que poderia realizá-la, de acordo com o contexto e a rotina da empresa. A Tabela 3 apresenta um exemplo desse relacionamento, sendo que as 3 primeiras colunas foram baseadas em Orci e Laryd [11] e a última coluna foi preenchida com as informações da empresa.

Após o preenchimento da Tabela 3, notou-se que alguns papéis do XS-CMM seriam acumulados por cargos da empresa, pois não havia cargos suficientes para cada papel. A Tabela 4 apresenta esse resultado parcialmente.

Com o mapeamento da Tabela 4 e o modelo de colaboração de Orci, Figura 1, gerou-se um modelo de colaboração de acordo com o contexto da empresa, apresentado na Figura 2, na qual os cargos da empresa estão em itálico. Adotou-se o padrão de linhas mais espessas para indicar uma alta colaboração e linhas tracejadas para indicar uma fraca colaboração. 
Tabela 3 - Relação entre o cargo da empresa que realiza a função associada ao papel do XS-CMM (baseado em ORCl e Laryd $[5,11]$ )

\begin{tabular}{|c|c|c|c|}
\hline $\begin{array}{c}\text { Papéis } \\
\text { XS-CMM }\end{array}$ & Funções no XS-CMM & $\begin{array}{c}\text { KPA/Prática- } \\
\text { chave }\end{array}$ & Cargos da empresa \\
\hline \multirow{2}{*}{$\begin{array}{c}\text { Gerente } \\
\text { Sênior }\end{array}$} & É responsável por uma política documentada para: & & \\
\cline { 2 - 4 } & - Gerenciamento de Requisitos & GR/C.1 & Gerente da U.N Web \\
\cline { 2 - 4 } & - Planejamento de Projeto & PPS/C.2 & Gerente U.N. Web \\
\multirow{2}{*}{$\begin{array}{c}\text { Gerente } \\
\text { Projeto }\end{array}$} & É responsável pelo gerenciamento de requisitos: & & \\
\cline { 2 - 5 } & - que a política da companhia seja seguida & GR/C.1 & Coord. Prodweb \\
\cline { 2 - 4 } & - revisa os requisitos alocados & GR/C.1 & Coord. Prodweb \\
\hline
\end{tabular}

Tabela 4 - Mapeamento dos cargos da empresa com os papéis do XS-CMM

\begin{tabular}{|l|l|}
\hline \multicolumn{1}{|c|}{ Cargos da empresa } & \multicolumn{1}{c|}{ Papéis do XS-CMM } \\
\hline Gerente da Unidade de Negócio de Web & Gerente Sênior \\
\hline \multirow{2}{*}{ Coordenador Prodweb } & Gerente de Software \\
\cline { 2 - 2 } & Gerente de Projeto \\
\cline { 2 - 2 } & Grupo de Gerenciamento de Configuração de Software \\
\hline \multirow{2}{*}{ Equipe de desenvolvimento } & Grupo de Engenharia de Software \\
\cline { 2 - 2 } & Grupo de Gerenciamento de Configuração de Software \\
\cline { 2 - 2 } & Grupo de teste
\end{tabular}

Tendo como exemplo a colaboração GP-ES, ela é alta, pois as atividades do Coordenador ProdWeb, quando no papel de Gerente de Projeto, são compartilhadas com um indivíduo da Equipe de Desenvolvimento no papel de membro do grupo de Engenharia de Software.

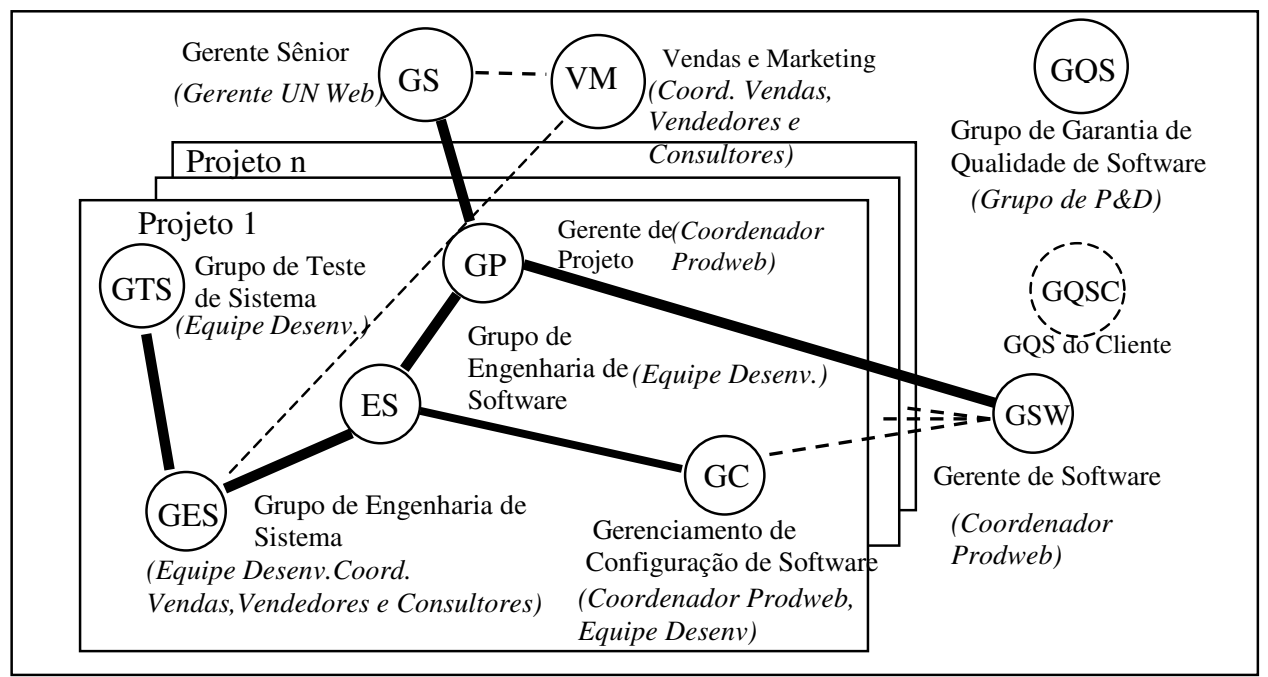

Figura 2 - Colaboração entre os papéis, segundo o contexto da empresa (adaptado de Orci [5])

Além da diferença no grau de colaboração existente na empresa (linhas espessas e linhas tracejadas) observaram-se também diferenças nas colaborações propostas na Figura 1. Um exemplo disso pode ser observado na Figura 2, na qual não há colaboração entre os papéis Gerente Sênior e Grupo de Engenharia de Software pois, na empresa, as atividades do primeiro são compartilhadas com o Gerente de Projeto. Esse é um fato que não pode ser deduzido a partir da Tabela 3, mas que foi observado no dia-adia da empresa e explicitamente declarado pelos indivíduos que assumem os respectivos 
cargos. A Figura 3 apresenta o mesmo mapeamento da Figura 2, mas sob o ponto de vista dos cargos da empresa.

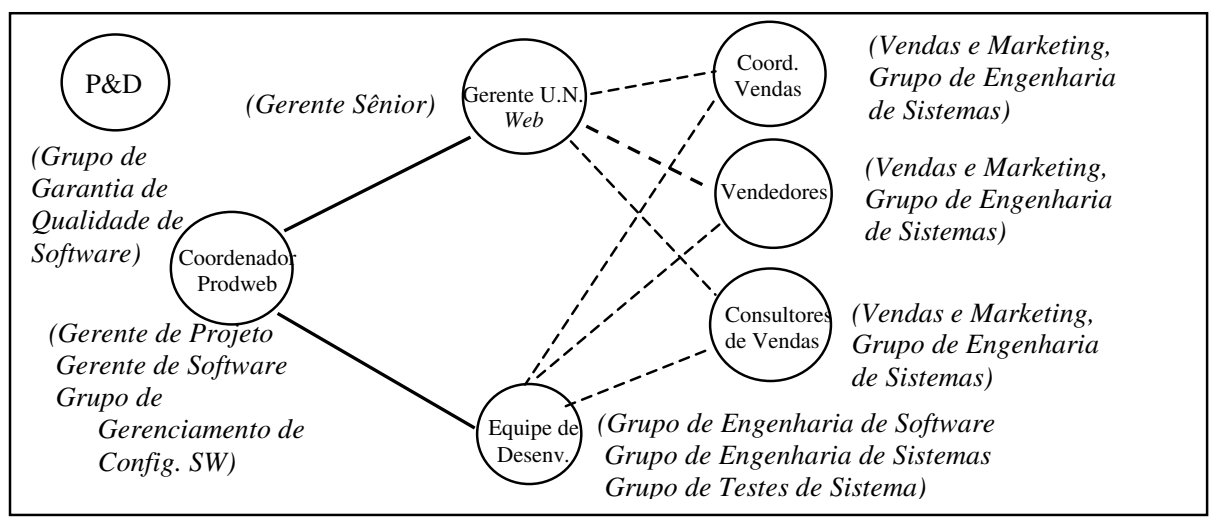

Figura 3 - Relacionamento dos Cargos da empresa com os papéis propostos por Orci

\section{Lições aprendidas}

- O fato da empresa em questão estar comprometida com questões de qualidade facilitou muito a realização do trabalho conduzido, as alterações de procedimentos, do processo, etc., o que é um ponto muito positivo e facilita o andamento das atividades e busca por novas alternativas.

- As pessoas envolvidas no processo de melhoria sempre acreditaram que os resultados obtidos seriam importantes para a empresa e sempre estiveram engajadas no processo, contribuindo, dessa forma, para sua evolução.

- O estudo sobre a adequação dos papéis do XS-CMM foi importante para a empresa pois, nesse modelo, obtiveram-se diretrizes para estabelecer as funções de cada funcionário envolvido no processo de desenvolvimento de software, o que permitiu com que a empresa visualizasse os cargos adequados aos papéis desse modelo.

- Mesmo sendo o modelo XS-CMM uma adaptação do SW-CMM para o porte da empresa em questão, observou-se que o contexto da empresa é determinante para sua utilização, o que resultou em uma nova adequação em algumas dessas adaptações propostas por Orci.

- A partir do momento que se conseguiu implantar duas melhorias, tendo a participação direta ou indireta das pessoas envolvidas no processo de software da empresa, notou-se que essas pessoas começaram a entender melhor a importância de um processo de melhoria e um grande entusiasmo em continuar com essas atividades.

\section{Conclusão}

O estudo da adequação dos papéis do XS-CMM foi importante, pois possibilitou à empresa obter uma visão de seus cargos em relação a esses papéis e também contribuiu com o mapeamento de responsabilidades dentro de seu processo. Como relatado, a proposta não foi seguida da forma que foi concebida, pois devido às particularidades da empresa, as colaborações entre os papéis não aconteciam de acordo com o modelo.

A melhoria do processo da empresa está baseada no XS-CMM e, junto com esse processo, buscam-se alternativas que contemplem, de forma mais adequada, a realidade de uma Empresa de Pequeno Porte. Apesar das grandes dificuldades encontradas 
durante as etapas deste trabalho, a empresa se mostrou satisfeita com os resultados obtidos até o momento. O grande desafio, a partir de agora, está na garantia de que as atividades relacionadas à melhoria de processo não serão paralisadas e que as não conformidades com as atribuições dos papéis não sejam fatores muito complicadores para esse processo.

\section{Referências Bibliográficas}

[1] PAULK, M. C. et al. "Capability Maturity Model for Software, Version 1.1", SEI Technical Report CMU/SEI-93-TR-24, 1993.

[2] PAULK, M. C. "Analyzing the Conceptual Relationship Between ISO/IEC 15504 (Software Process Assessment) and the Capability Maturity Model for Software". 1999. In : Proceedings International Conference on Software Quality.

[3] PAULK, M.C., "Using the Software CMM With Good Judgment", ASQ Software Quality Professional, Vol. 1, No. 3, June 1999, pp. 19-29.

[4] JOHNSON, D. L. and BRODMAN, J. G., "Tailoring the CMM form Small Business, Small Organizations, and Small Project", Software Process Newsletter, Committee on Software Process, IEEE Computer Society, No. 8, Winter 1997.

[5] ORCI, Terttu, "Capability Maturity Model for Extra Small Organizations Level 2", Technical Report UMINF 00.13, Department of Computing Sciences, Umeå University, $\quad 2000 . \quad$ Deptember em: $<$ http://www.cs.umu.se/ jubo/Projects/QMSE/>, Acesso em: 25 jun. 2002

[6] ORCI, Terttu, "Capability Maturity Model for Extra Extra Small Organizations Level 2”, Technical Report UMINF 00.12, Department of Computing Sciences, Umeå University, September 2000. Disponível em: $<$ http://www.cs.umu.se/ jubo/Projects/QMSE/>, Acesso em: 25 jun. 2002

[7] ORCI, Terttu, "Capability Maturity Model for Small Organizations Level 2", Technical Report UMINF 00.14, Department of Computing Sciences, Umeå University, $2000 . \quad$ Deptember em: $<$ http://www.cs.umu.se/ jubo/Projects/QMSE/>, Acesso em: 25 jun. 2002

[8] SEBRAE. Disponível em <http://www.sebrae.org.br/br/ued/introducao.htm>. Acesso em 1 de junho de 2004

[9] ORCI, Terttu and LARYD, Astrid "Dynamic CMM for Small Organizations Implementation Aspects". In: Proceedings Spi2000, The International Conference on Software Process Improvement Conference, 2000,Gothenburg, Sweden. Disponível em: <http://www.cs.umu.se/ jubo/Projects/QMSE/>. Acesso em: 25 jun. 2002.

[10] ORCI, Terttu, LARYD, Astrid; BÖRSTLER Jürgen, "CMM for Small Organizations, Level 2 - The Supplements”, Technical Report UMINF 01.19, Department of Computing Science, Umeå University, 2001. Disponível em: $<$ http://www.cs.umu.se/ jubo/Projects/QMSE/>. Acesso em: 25 de junho de 2002.

[11] ORCI, Terttu and LARYD, Astrid "CMM for Small Organizations", Technical Report UMINF 00.20, Department of Computing Science, Umeå University, 2000. Disponível em: <http://www.cs.umu.se/ jubo/Projects/QMSE/>. Acesso em: 25 jun. 2002.

[12] KELLY, D.P.; CULLETON, B.; "Process improvement for small organizations". IEEE Computer, Volume: 32 Issue: 10 , October 1999, pp 41 -47.

[13] TAVARES, D. P. D., "Melhoria de Processo na Pequena Empresa : um estudo de caso”. 2002. 96f. Dissertação (Mestrado em Engenharia de Software) - Centro de Ciências Exatas e de Tecnologia, Universidade Federal de São Carlos, São Carlos. 